\title{
The visual perception of length along intrinsically curved surfaces
}

\author{
J. FARLEY NORMAN, HIDEKO F. NORMAN, YOUNG-LIM LEE, and DASHA STOCKTON \\ Western Kentucky University, Bowling Green, Kentucky \\ and \\ JOSEPH S. LAPPIN \\ Vanderbilt University, Nashville, Tennessee
}

\begin{abstract}
The ability of observers to perceive three-dimensional (3-D) distances or lengths along intrinsically curved surfaces was investigated in three experiments. Three physically curved surfaces were used: convex and/or concave hemispheres (Experiments 1 and 3) and a hyperbolic paraboloid (Experiment 2). The first two experiments employed a visual length-matching task, but in the final experiment the observers estimated the surface lengths motorically by varying the separation between their two index fingers. In general, the observers' judgments of surface length in both tasks (perceptual vs. motoric matching) were very precise but were not necessarily accurate. Large individual differences (overestimation, underestimation, etc.) in the perception of length occurred. There were also significant effects of viewing distance, type of surface, and orientation of the spatial intervals on the observers' judgments of surface length. The individual differences and failures of perceptual constancy that were obtained indicate that there is no single relationship between physical and perceived distances on 3-D surfaces that is consistent across observers.
\end{abstract}

Interest in how human observers perceive the length of spatial intervals dates to antiquity (e.g., Aristotle, trans. 1984) and was maintained in the Renaissance (Descartes, 1628/1955). Indeed, many of the earliest psychophysicists and psychologists of the 19th century (Weber, Fechner, Helmholtz, Wundt, etc.) were interested in the perception and discrimination of length. For example, Weber (1834/1978) initiated the field of psychophysics by showing that human observers can discriminate differences in length very precisely. In his pioneering experiments, he found that small differences in length, on the order of $1 \%-5 \%$, were reliably detectable. Early in his career (17 years before the formal founding of the first "psychological laboratory"), Wundt (1862, as cited by Graham, 1951) pointed out that human observers tended to overestimate vertical lengths and perceive them as being longer than physically equivalent horizontal lengths (i.e., the now well-known horizontalvertical illusion). Indeed, many of the famous geometrical illusions involve distortions in the perception of length (e.g., the Müller-Lyer and Sander illusions; see Woodworth \& Schlosberg, 1954). In the 1960s, Julesz

Portions of this research were presented at the 2002 annual meeting of the Vision Sciences Society, Sarasota, FL. Correspondence concerning this article should be addressed to J. F. Norman, Department of Psychology, Western Kentucky University, Bowling Green, KY 42101 (e-mail: farley.norman@wku.edu).

Note-This article was accepted by the previous editorial team, headed by Neil Macmillan.
(1971) incorporated geometrical figures such as the Müller-Lyer in random-dot stereograms (with no monocular contours, the figure was defined only by binocular disparity), and the typical illusion occurred. This result shows that the perceptual distortion of length caused by such figures is the result of physiological mechanisms in the cerebral cortex (since information from the left and the right eyes is kept separate until the level of the primary visual cortex).

Since the 19th century, researchers have required observers to judge distances or lengths between points or objects in empty space (Gogel, 1960; Norman \& Todd, 1998; Norman, Todd, Perotti, \& Tittle, 1996), between locations on a ground plane (Gilinsky, 1951; Loomis, Da Silva, Fujita, \& Fukusima, 1992; Wagner, 1985), between locations on a flat plane or tabletop (Baird \& Biersdorf, 1967; Norman, Lappin, \& Norman, 2000), or between separate locations on the surface of a singly curved solid object, such as a cylinder (Norman et al., 2000). This past research has shown that in many circumstances, perceived distances in depth are foreshortened and appear smaller than their actual distances (e.g., Gilinsky, 1951; Norman et al., 1996; Wagner, 1985). In contrast, Norman et al. (2000) found that distances or lengths in depth between surface locations on a cylinder were perceptually overestimated and appeared larger than they really were.

The results of previous research clearly demonstrate that although observers' perceptions of three-dimensional (3-D) length may be precise (judgments of length are typically very reliable, with low variability over time), they are often inaccurate and that significant over- and 
underestimations occur. One unresolved question concerns perceptions of length along the surfaces of more complicated objects that possess curvature in many directions. Norman et al. (2000) found that observers frequently overestimated the lengths of spatial intervals that lay along the curvature of a cylinder in depth. But is this result general and representative of the perception of length on more ordinary surfaces? Almost all natural objects have curvatures in many different directions, unlike simple objects such as cylinders (e.g., consider rocks, vegetables, fruits, etc.). Indeed, it has been mathematically proven (see Hilbert \& Cohn-Vossen, 1952; Koenderink, 1990; Koenderink \& van Doorn, 1982) that all smoothly curved object surfaces are composed of two generic types of surface regions, where the sign of surface curvature is the same in perpendicular directions (such as a convex or concave hemisphere) or is the $o p$ posite in perpendicular directions (like a "saddle"). These two generic surface regions are separated by a line of points where the surface is locally shaped like a cylinder (i.e., parabolic points). The purpose of the present study was to assess the precision and accuracy of observers' perceptions of length along hemispherical and saddle-shaped surfaces, since the surfaces of naturally curved objects are composed primarily of these two types of surface regions. In the present set of experiments, the perception of length along hemispherical surfaces was explored in Experiment 1, whereas Experiment 2 was designed to evaluate the perception of length along saddleshaped surfaces. A visual-matching task was employed in Experiments 1 and 2; in contrast, the observers performed a motor response task in Experiment 3 in order to estimate the magnitude of the surface lengths.

\section{EXPERIMENT 1}

\section{Method}

Stimulus displays. Convex and concave hemispherical surfaces, both with a radius of curvature of $14 \mathrm{~cm}$, were created out of Plaster of Paris/gypsum ( $\mathrm{CaSO}_{4}-2 \mathrm{H}_{2} \mathrm{O}$-calcium sulfate dihydrate). The surfaces were covered with a "Jackson Pollock" type of texture (see Pollock, 1978). The presence of texture served a variety of purposes. First, the texture covering the curved surfaces created texture gradients of size, shape, and density in the observers' retinal images, and these gradients are themselves known to be effective sources of optical information about 3-D shape. Second, the texture served as a carrier for binocular disparity, which is one of the most powerful sources of information about object shape. Four colors were used in creating the textures: red, white, silver, and teal. Since the convex and concave hemispheres were illuminated from above using standard fluorescent light bulbs, image shading was also present in the observers' retinal images; the patterns of image shading produced were different for the convex and the concave hemispheres, due to their different distributions of surface orientations. It should be readily apparent that the viewing situation in this experiment was full cue: The observers had access to a wealth of optical information to support their perception of the 3-D shape of the objects, as well as the lengths of spatial intervals along their surfaces. Grayscale photographs of the two hemispherical surfaces are shown in Figure 1.

For each object, 24 surface locations were chosen at random. Light-emitting diodes (LEDs) were then embedded into the object surfaces at those locations. From these 24 surface locations, 40 pairs of LEDs, defining distances to be judged, were then chosen from all possible pairs (276 possible pairs, 24 things taken 2 at a time). Ten of the distances were oriented along the object surfaces so that they were vertically aligned in the frontoparallel plane, whereas another 10 distances had a horizontal alignment. The remaining 20 lengths were oriented so that they had diagonal orientations (both clockwise [CW] and counterclockwise [CCW], at approximately $45^{\circ}$ from a vertical orientation) in the frontoparallel plane. The surfaces were viewed by the observers at near $(50 \mathrm{~cm})$ and far $(180 \mathrm{~cm})$ viewing distances.

Procedure. The LEDs def ining the surface lengths or distances were controlled by a Dell Dimension XPS T450 computer using a Data Translation DT-335 digital output board. On every trial, the computer would highlight a pair of LEDs, and the observer would be asked to adjust the length of a line segment presented on a NEC 22-in. color monitor (located at a middle distance of $100 \mathrm{~cm}$ ) until its length matched the length on the curved surface between the two highlighted LEDs. The observers were required to judge the length along the curved object's surface between the highlighted LEDs. In any given experimental session, each observer judged all of the 40 surface lengths two times at either the near or the far viewing distance ( 80 trials for each experimental session). The observers participated in five experimental sessions at each viewing distance. Thus, at the end of the experiment, the observers had performed 800 judgments for each surface. Of the 5 observers who judged lengths on the convex hemisphere, 2 viewed the object from the near distance first, whereas the remaining 3 used the far viewing distance first. Three observers performed the task at the near distance first for the concave hemisphere, whereas the remaining 2 estimated the lengths from the far distance first.

Observers. There was a total of 7 observers, who were students or faculty at Western Kentucky University. Three of the observers, all coauthors (H.F.N., Y.L., and D.S.) estimated the surface lengths for both the convex and the concave hemispheres. Two observers (K.D. and E.D.) judged lengths along the convex hemisphere, whereas 2 other observers (L.M.H. and J.R.L.) judged lengths along the concave hemisphere. The 4 observers who were not coauthors (K.D., E.D., L.M.H., and J.R.L.) had no previous experience as psychophysical observers and were naive with regards to the purposes of the experiment. All the observers had normal or correctedto-normal visual acuity.

\section{Results and Discussion}

The results for two representative observers (H.F.N. and E.D.) for the convex hemisphere are shown in Figure 2. The data for all 5 observers for this object are presented in Table 1, as slopes of the best-fitting regression lines indicating the relationship between perceived length and actual length. Figure 2 shows the results of one observer (H.F.N.) who consistently overestimated the surface lengths and perceived them as being much longer than they actually were (by $30 \%-50 \%$ ). Observer Y.L.'s results depict a similar pattern. The other observer's (E.D.'s) judgments that are shown in Figure 2 were accurate at the near distance but were less accurate at the far distance. At the far distance, this observer often underestimated the surface lengths and perceived them as being up to $17 \%$ shorter than they actually were. The remaining observers' (K.D. and D.S.) results fell in between these two patterns: K.D. overestimated the surface lengths by smaller amounts than did either H.F.N. or Y.L. $(5 \%-19 \%)$, whereas the judgments of D.S. were generally accurate, with the slopes of the best-fitting regression lines being near 1.0. 


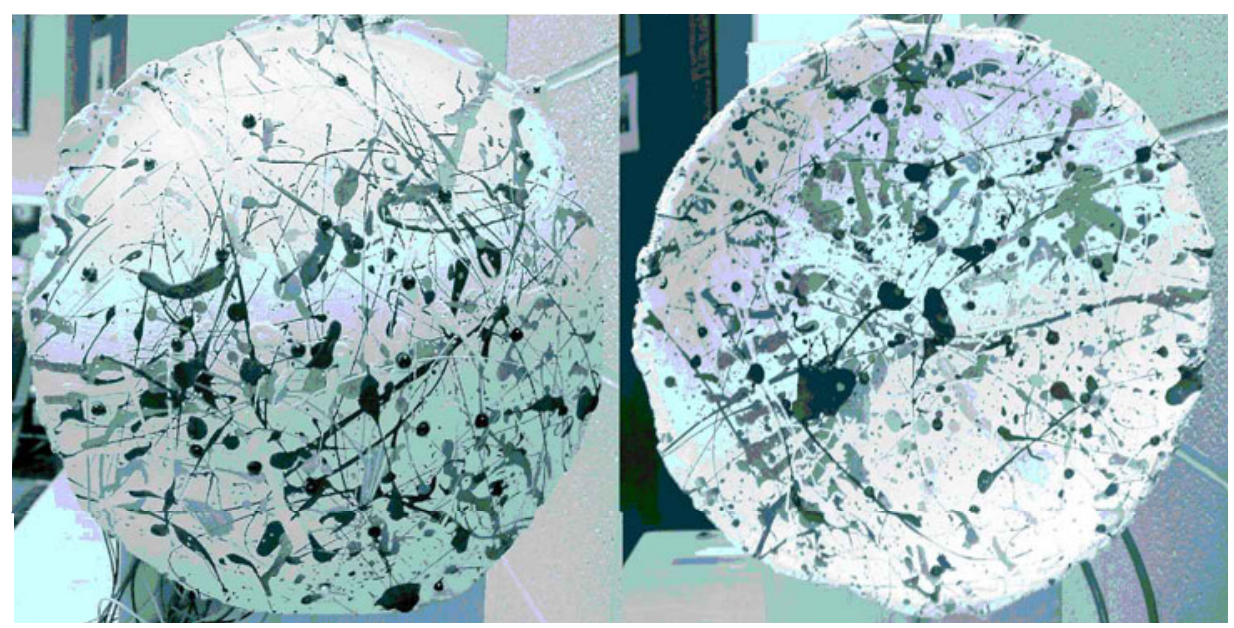

Figure 1. Photographs of the convex (left panel) and concave (right panel) hemispheres used as stimuli in Experiment 1.

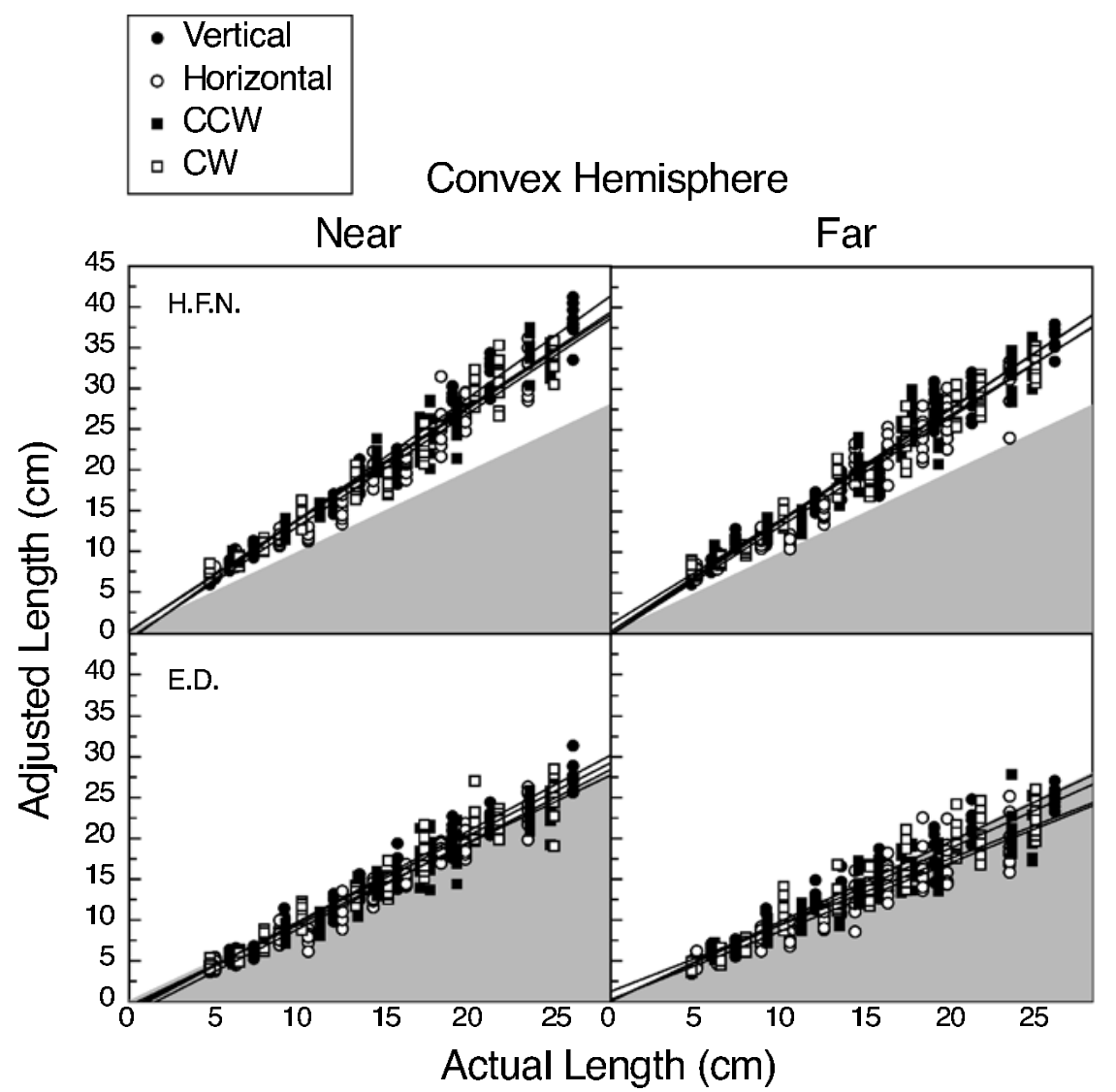

Figure 2. Results of Experiment 1 for the convex hemisphere for two representative observers (H.F.N. and E.D.). The observers' perceived lengths are plotted as functions of the actual surface lengths. In each graph, the boundary between the white and the gray areas indicates a slope of 1.0. Therefore, data points that fall within the white regions indicate overestimation, whereas data points that are plotted within the gray regions indicate underestimation. CW, clockwise; CCW, counterclockwise. 
Table 1

Slopes of Best-Fitting Regression Lines for the Convex Hemisphere

\begin{tabular}{lcccc}
\hline & \multicolumn{4}{c}{ Length Orientation } \\
\cline { 2 - 5 } Observer & $\mathrm{V}$ & $\mathrm{H}$ & $\mathrm{CCW}$ & $\mathrm{CW}$ \\
\hline \multicolumn{5}{c}{ Near Distance } \\
H.F.N. & 1.513 & 1.405 & 1.400 & 1.390 \\
Y.L. & 1.466 & 1.446 & 1.364 & 1.389 \\
D.S. & 1.122 & 1.089 & 0.952 & 0.997 \\
E.D. & 1.123 & 1.076 & 1.010 & 1.077 \\
K.D. & 1.188 & 1.116 & 1.063 & 1.078 \\
& \multicolumn{5}{c}{ Far Distance } \\
H.F.N. & 1.397 & 1.350 & 1.387 & 1.301 \\
Y.L. & 1.278 & 1.399 & 1.185 & 1.274 \\
D.S. & 1.058 & 1.024 & 0.939 & 0.929 \\
E.D. & 0.992 & 0.849 & 0.826 & 0.945 \\
K.D. & 1.140 & 1.071 & 1.103 & 1.054 \\
\hline
\end{tabular}

Note- $\mathrm{V}$, vertical; H, horizontal; CCW, counterclockwise; CW, clockwise.

The results shown in Figure 2 and Table 1 clearly indicate that, in general, the observers' judgments of surface length along the convex hemisphere were not accurate (there were significant overestimations, underestimations, etc.). However, all of the observers were stable in their individual patterns over time, and their judgments were precise. The average Pearson $r$ correlation coefficient (correlating the observers' perceived lengths with the actual surface lengths) across all the observers and conditions was .97; therefore, fully $94 \%$ of the variance in the observers' perceived lengths could be accounted for by the variations in the actual surface lengths. Another way to show the observers' precision is in terms of reliability, which we calculated as the standard deviation of the observers' 10 judgments for each condition divided by the mean. The overall reliabilities for the observers ranged from a low of $6.3 \%$ (H.F.N., most reliable) to a high of $11.3 \%$ (K.D., least reliable); these reliability values did not vary significantly with any of the experimental manipulations and are consistent with those from earlier studies of the perception of length (Norman et al., 2000; Norman et al., 1996).

The data shown in Table 1 were subjected to a two-way within-subjects analysis of variance (ANOVA). The results of the ANOVA revealed a significant effect of surface length orientation, so that surface lengths oriented in a vertical direction in the observers' projected retinal images were perceived to be longer than physically equivalent horizontal lengths $[F(3,12)=7.354, p=.005]$. On average, the vertical lengths were perceived as being longer than physically equivalent horizontal lengths by $3.8 \%$. This effect of length orientation is approximately half that involved in the conventional horizontal-vertical illusion (which was 7.2\% in Finger \& Spelt, 1947).

The results of the ANOVA also revealed a significant effect of viewing distance, so that the same surface lengths appeared longer at the near viewing distance and shorter at the far viewing distance $[F(1,4)=10.496, p=$ $.032]$. On average, the surface lengths were perceived to be $7.8 \%$ longer at the near distance, and all 5 observers showed this trend. It is important to keep in mind, however, that both the near and the far viewing distances fell within the near range of visual space in which stereopsis is effective, and our observers viewed these surfaces in fullcue conditions with both eyes. In addition, the observers were free to move their heads and generate monocular motion parallax, which is a rich source of information about distance and 3-D shape in its own right (Rogers \& Graham, 1979). Nevertheless, there were significant over- and underestimations, as well as systematic effects of viewing distance and orientation of the surface lengths.

The results for the concave hemisphere are shown in Figure 3 (2 representative observers, L.M.H. and D.S.) and Table 2 (all the observers). Once again, there were large individual differences in the perception of surface lengths: L.M.H. and H.F.N. overestimated by amounts ranging from $30 \%$ to $53 \%$, whereas the judgments of Y.L. and D.S. were more accurate. J.R.L.'s judgments were generally accurate at the near distance, but at the far distance she overestimated the surface lengths by amounts up to $20 \%$. Of the 3 observers who judged both the convex and the concave hemispheres, H.F.N. and D.S. showed essentially the same pattern of results for both surfaces: H.F.N. always tended to overestimate by large amounts, whereas the judgments of D.S. were generally accurate. The remaining observer's (Y.L.'s) judgments had different patterns for lengths judged along the convex and the concave hemispheres. For the convex hemisphere, she overestimated by large amounts (similar to H.F.N.), but her judgments were relatively accurate for lengths presented along the surface of the concave hemisphere (similar to D.S.). After completion of the data collection, she (Y.L.) informally reported that the concave hemisphere appeared to have less curvature and less front-to-back depth than did the convex hemisphere (i.e., it appeared "flatter"); this informal report is consistent with smaller judged lengths on the concave hemisphere. This is an interesting result, because the concave hemisphere had exactly the same depths, curvatures, and other physical dimensions as the convex hemisphere; yet at least for this observer, it was less effective in producing perceptions of "depthfullness" and curvature.

The observers were just as consistent in their judgments of surface length along the concave hemisphere as in those for the convex hemisphere. The average Pearson $r$ correlation coefficient between the observers' perceived lengths and the actual surface lengths was .98 ; therefore, $96 \%$ of the variance in the observers' perceived lengths could be accounted for by the variability in the actual surface lengths. Also like the earlier judgments along the convex hemisphere, the observers were very reliable over time in estimating the surface lengths along the concave hemisphere. The overall reliabilities for this surface ranged from $4.8 \%$ (H.F.N., most reliable) to $8.5 \%$ (J.R.L., least reliable).

The results depicted in Table 2 were also subjected to a two-way within-subjects ANOVA. Like the results for the convex hemisphere, the ANOVA for the concave hemisphere revealed a significant effect of surface length ori- 

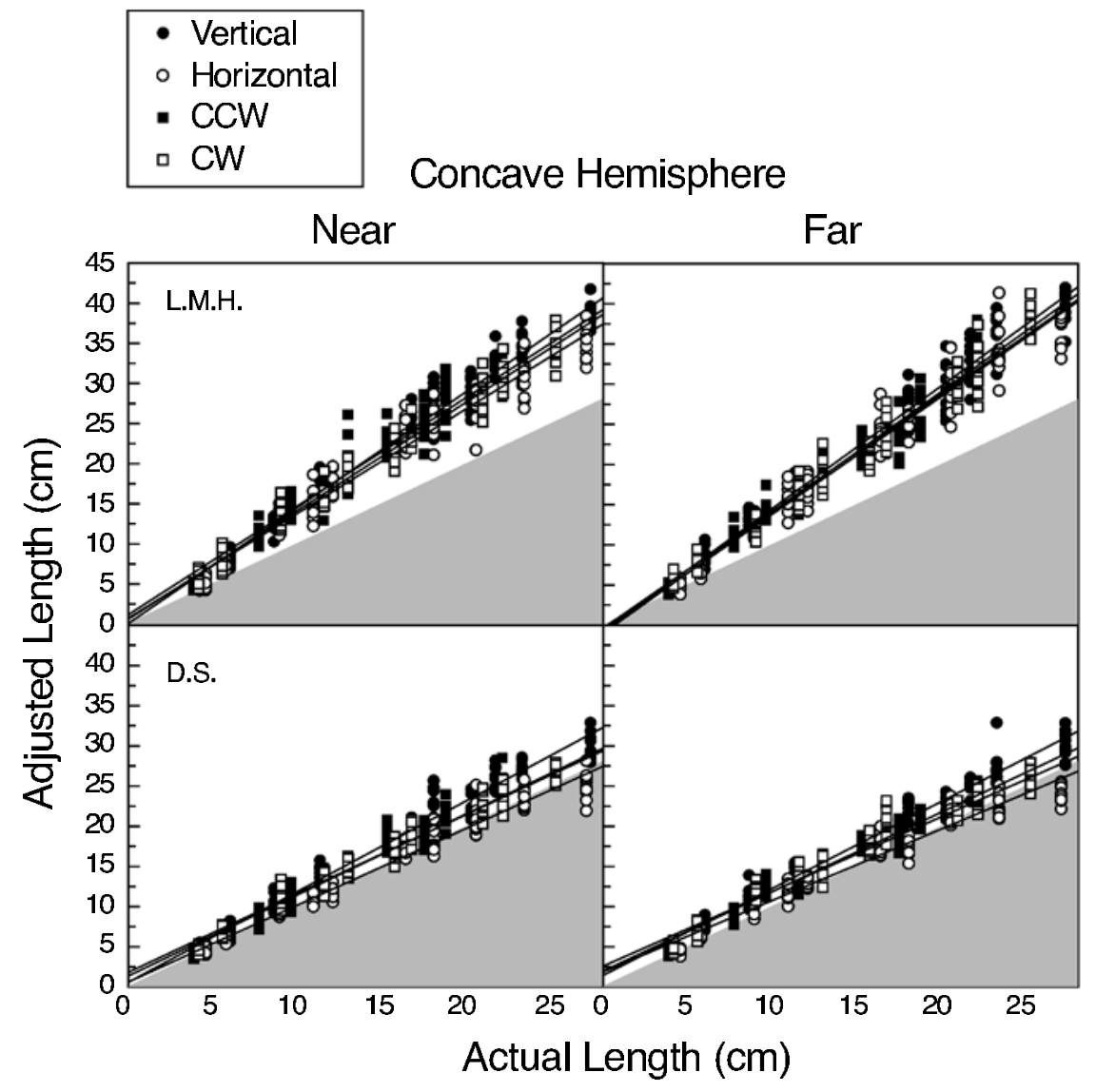

Figure 3. Results of Experiment 1 for the concave hemisphere for two representative observers (L.M.H. and D.S.). The observers' perceived lengths are plotted as functions of the actual surface lengths. In each graph, the boundary between the white and the gray areas indicates a slope of 1.0. Therefore, data points that fall within the white regions indicate overestimation, whereas data points that are plotted within the gray regions indicate underestimation. CW, clockwise; CCW, counterclockwise.

entation, so that the vertically oriented lengths appeared to be longer than physically equivalent horizontal lengths $[F(3,12)=14.1, p=.0003]$. On average, the surface lengths aligned along a vertical direction were perceived by the observers to be $9.1 \%$ longer than the lengths aligned in a horizontal direction. The magnitude of this horizontalvertical anisotropy is even larger than the conventional horizontal-vertical illusion, which was found to be $7.2 \%$ by Finger and Spelt (1947). Unlike the results for the convex hemisphere, the ANOVA conducted upon the observers' judgments of length along the concave hemisphere did not reveal an overall or main effect of viewing distance $[F(1,4)=0.4, p>.05]$. However, a conclusion that viewing distance did not affect the observers' perceptions of length in this case would be misleading, because different observers showed different effects of viewing distance for the concave hemisphere. These individual effects of viewing distance for the concave hemisphere are shown in Table 3. In this table, a $t$ test was conducted for each individual observer (dependent-samples $t$ test), using the results for each of the five experimental sessions as repli- cations. The results shown in Table 3 clearly indicate effects of viewing distance for the majority of the observers: The perceived surface lengths for L.M.H. and J.R.L. increased significantly as viewing distance was increased, whereas those for Y.L. decreased. Similar conflicting effects of viewing distance (changes in viewing distance produce opposite effects for different observers) on observers' perceptions of surface length have been obtained in previous studies in which simpler cylindrical surfaces have been used (e.g., Norman et al., 2000).

\section{EXPERIMENT 2}

The results of Experiment 1 showed that observers' perceptions of length along surfaces with positive Gaussian curvature (i.e., convex and concave hemispheres) are precise but not necessarily accurate. The results also showed that the perception of surface length is influenced by changes in viewing distance and orientation of the lengths on the surface. The purpose of Experiment 2 was to similarly investigate the human perception of length 
Table 2

Slopes of Best-Fitting Regression Lines for the Concave Hemisphere

\begin{tabular}{lcccc}
\hline & \multicolumn{4}{c}{ Length Orientation } \\
\cline { 2 - 5 } Observer & $\mathrm{V}$ & $\mathrm{H}$ & $\mathrm{CCW}$ & $\mathrm{CW}$ \\
\hline \multicolumn{5}{c}{ Near Distance } \\
H.F.N. & 1.410 & 1.330 & 1.331 & 1.303 \\
Y.L. & 1.056 & 1.039 & 1.016 & 1.012 \\
D.S. & 1.133 & 0.959 & 0.985 & 1.013 \\
L.M.H. & 1.415 & 1.310 & 1.359 & 1.358 \\
J.R.L. & 1.065 & 0.936 & 0.946 & 0.981 \\
& \multicolumn{5}{c}{ Far Distance } \\
H.F.N. & 1.438 & 1.337 & 1.327 & 1.287 \\
Y.L. & 0.989 & 0.943 & 0.908 & 0.933 \\
D.S. & 1.085 & 0.891 & 0.932 & 0.991 \\
L.M.H. & 1.529 & 1.469 & 1.461 & 1.508 \\
J.R.L. & 1.209 & 1.127 & 1.065 & 1.173 \\
\hline
\end{tabular}

Note-V, vertical; H, horizontal; CCW, counterclockwise; CW, clockwise.

along a qualitatively different curved surface, one with negative Gaussian curvature. This is important, since Koenderink and van Doorn (1982; see also Koenderink, 1990) have shown that all smooth bidirectionally curved object surfaces can be described or decomposed into surface regions with positive and negative Gaussian curvature.

\section{Method}

Stimulus displays. A hyperbolic paraboloid with a radius of curvature of $14 \mathrm{~cm}$ (same magnitude of curvature as the convex and concave hemispheres used in Experiment 1) was created, using the same materials as the surfaces used in Experiment 1 (Plaster of Paris/gypsum). The surface was once again covered with a fourcolor "Jackson Pollock" texture to provide texture gradients in the observers' retinal images, and the texture also served as a carrier for binocular disparity and/or monocular motion parallax. A photograph of the hyperbolic paraboloid is shown in Figure 4.

Twenty-four surface locations on the hyperbolic paraboloid were once again selected at random. LEDs were embedded into the surface at those locations. As in Experiment 1, 40 surface lengths were chosen to be investigated (10 lengths in four different orientations: horizontal, vertical, oblique-CW, and oblique-CCW). The hyperbolic paraboloid was positioned at the same viewing distances as those used in Experiment $1(50$ and $180 \mathrm{~cm})$.

Procedure. The experimental procedures and the task were identical to those in Experiment 1. On any given trial, the observers estimated the length between two highlighted LEDs along the curved surface of the hyperbolic paraboloid. Each observer judged all of the 40 surface lengths for a total of 10 times ( 2 trials per length $\times$ 5 experimental sessions) at each of the two viewing distances. Thus, each observer performed 800 judgments of the surface lengths on the hyperbolic paraboloid.

Observers. There was a total of 5 observers, who were students or faculty at Western Kentucky University or Vanderbilt University. Four of the 5 observers had participated in Experiment 1 (J.S.L. did not judge lengths for either the convex or the concave hemisphere). E.D. was naive with regards to the purposes of the experiment. All the observers had normal or corrected-to-normal visual acuity.

\section{Results and Discussion}

The complete results for 2 observers (H.F.N. and J.S.L.) are shown in Figure 5. Summaries of the other observers' results are provided in Table 4. Overall, the results for the hyperbolic paraboloid are very similar to those ob- tained for the hemispherical surfaces in Experiment 1: Some of the observers (H.F.N. and Y.L.) overestimated the surface lengths (by 23\%-39\%), whereas other observers (E.D. and J.S.L.) often showed patterns of underestimation (by 5\%-33\%). The results for J.S.L. and D.S. are especially interesting. Their results were qualitatively different at the two different viewing distances: They generally underestimated the surface lengths at the near distance but overestimated the same lengths at the far distance.

The observers' judgments in this experiment were precise and reliable, even if they were often not accurate. The average Pearson $r$ correlation coefficient across all conditions, lengths, and observers was .98. The observers' average individual reliabilities (standard deviations of repeated judgments for single experimental conditions divided by the mean of the judgments) ranged from a low of $5.2 \%$ (H.F.N., most reliable) to a high of $8.7 \%$ (E.D., least reliable). These values are comparable to those obtained in Experiment 1 and demonstrate that human observers are equally reliable at judging lengths upon surfaces with positive and negative Gaussian curvatures.

The data depicted in Table 4 were subjected to a twoway within-subjects ANOVA. The results of the ANOVA revealed a significant effect of the orientation of the surface lengths in the observers' retinal images $[F(3,12)=$ $3.721, p=.042$ ]. On average, the observers perceived the vertically oriented lengths to be $12.9 \%$ longer than physically identical horizontal lengths. This effect of length orientation is even larger than the conventional horizontal-vertical illusion (Finger \& Spelt, 1947).

The effects of viewing distance were similar to those observed for the concave hemisphere in Experiment 1. The results of the two-way ANOVA did not reveal a significant main effect of distance $[F(1,4)=1.15, p>.05]$, but significant and opposite effects of distance were obtained when the observers' individual results were analyzed (see Table 5). A majority of the observers showed effects of viewing distance. Two observers (J.S.L. and D.S.) perceived the surface lengths to be significantly longer at the far distance, whereas E.D. perceived the lengths to be shorter at the far distance.

\section{EXPERIMENT 3}

The results of Experiments 1 and 2 showed that observers' visual perceptions of length along curved surfaces

Table 3

Effects of Distance for the Concave Hemisphere

\begin{tabular}{|c|c|c|c|c|c|}
\hline \multirow[b]{2}{*}{ Observer } & \multicolumn{2}{|c|}{ Slope } & \multirow[b]{2}{*}{ Change $\%$} & \multirow[b]{2}{*}{$t$} & \multirow[b]{2}{*}{$p$} \\
\hline & $\begin{array}{c}\text { Near } \\
\text { Judgments }\end{array}$ & $\begin{array}{c}\text { Far } \\
\text { Judgments }\end{array}$ & & & \\
\hline H.F.N. & 1.349 & 1.357 & +0.6 & -0.614 & .572 \\
\hline Y.L. & 1.034 & 0.949 & -8.2 & 15.676 & $<.0001$ \\
\hline D.S. & 1.031 & 0.982 & -4.8 & 1.590 & .187 \\
\hline L.M.H. & 1.376 & 1.495 & +8.6 & -4.997 & .008 \\
\hline J.R.L. & 0.988 & 1.149 & +16.3 & -6.474 & .003 \\
\hline
\end{tabular}

Note-Results of the regression analysis that evaluated the effects of viewing distance for the concave hemispherical surface used in Experiment 1. All $d f \mathrm{~s}=4$. 


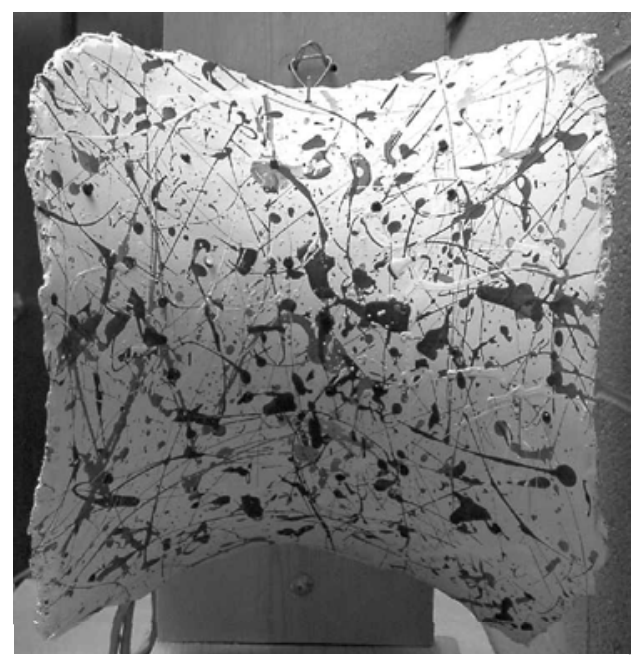

Figure 4. Photograph of the saddle-shaped hyperbolic paraboloid used as the stimulus in Experiment 2. As can be seen in the figure, the surface was oriented so that the concave curvatures were oriented horizontally, whereas the convex curvatures occurred in a vertical direction.

are often inaccurate; our observers frequently over- or underestimated the depicted surface lengths by amounts up to $50 \%$. These patterns of perceptual distortion were reliable for each individual observer and persisted over many experimental sessions. Both previous experiments used a visual-matching paradigm in which the observers adjusted the length of one stimulus until its length appeared to visually match that of a curved spatial interval along the surface of either a hemisphere (Experiment 1) or a hyperbolic paraboloid (Experiment 2). Over the past 10-15 years, there have been several instances in which a task that apparently required a knowledge of distance could not accurately be performed on the basis of an observer's conscious visual experience but could be performed if the observer made his or her judgments motorically. For example, Loomis et al. (1992) found that when making visual judgments of distance in an outdoor environment, their observers typically showed significant patterns of underestimation (similar to the underestimations produced by some of the observers in our experiments). Nevertheless, if their observers were asked to look at a "target" in depth, estimate its distance, and then walk blindfolded to it, they were usually able to perform this task with no evidence of a systematic compression or underestimation of perceived distances in depth. Might this pattern of results occur also when observers are asked to estimate the extents of spatial intervals along the surfaces of curved objects? In the present experiment, we evaluated this possibility.

\section{Method}

Stimulus displays. The convex hemisphere that was used in Experiment 1 was also employed in this experiment. The observers estimated the lengths of the same set of vertically oriented intervals investigated in Experiment 1. The observers viewed the surface from the same near distance $(50 \mathrm{~cm})$ as that used in Experiment 1 .

Procedure. The experimental procedures were similar to those used in Experiments 1 and 2. The only difference was in terms of how the observers made their length-matching adjustment. In this experiment, the observers were asked to estimate the length between any given pair of highlighted LEDs along the surface of the hemisphere and then to adjust the separation between their two index fingers (starting from zero separation) until the magnitude of that separation matched the length of the depicted surface interval. The observers were allowed to increase and decrease the separation between their two index fingers until they were satisfied with their matching adjustment. It is important to note that the observers were not allowed to look at their fingers (or hands or arms) while performing this judgment; the observers continued to visually fixate the curved surface intervals while motorically matching their lengths. When the observers were satisfied with their judgment on each trial, they closed their eyes, and the distance between their two index fingers was then measured by an experimenter (since the observers' eyes were closed during the measurement of their finger separation, the observers never received any feedback regarding their performance during the experiment).

Each observer judged the 10 vertically oriented surface lengths a total of 10 times ( 5 trials per length $\times 2$ experimental sessions). Thus, each observer performed a total of 100 motor judgments of the surface lengths on the convex hemisphere.

Observers. Three observers who had participated in Experiments 1 and 2 (H.F.N., Y.L., and D.S.) also served as subjects in the present experiment. All of the observers had normal or correctedto-normal visual acuity.

\section{Results and Discussion}

The results for all 3 observers are shown in Figure 6. The data for the comparable visual judgments are also plotted for comparison. It is readily apparent that the observers' patterns of results for estimating the surface lengths motorically are essentially identical to their visualmatching performances obtained in Experiment 1. H.F.N. and Y.L. once again showed large overestimations (the slopes of the best-fitting regression lines were 1.719 and 1.636, respectively). Just as in Experiment 1, the results for D.S. were more accurate. However, this observer still overestimated the surface lengths by about $14 \%$ (i.e., the slope of the best-fitting regression line was 1.140$)$. The results of a $t$ test for dependent samples revealed that there was no significant difference between the observers' motoric-matching adjustments in the present experiment and the visual-matching judgments obtained in Experiment $1[t(2)=2.28, p>.05]$. However, all 3 of the observers overestimated more in the motoric-matching conditions than in the equivalent visual-matching conditions. The failure to find a significant difference was probably due to low sample size.

The observers were reliable and precise in their motoric estimates of perceived surface length. The average Pearson $r$ correlation coefficient (correlation between actual surface lengths and perceived surface lengths) was .97, the same as that found for the visual-matching judgments of the convex hemisphere in Experiment 1 . The observers' average reliability (standard deviation of repeated adjustments for single conditions divided by 


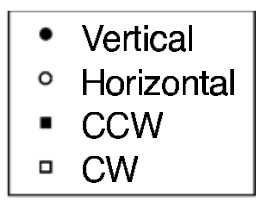

\section{Hyperbolic paraboloid}

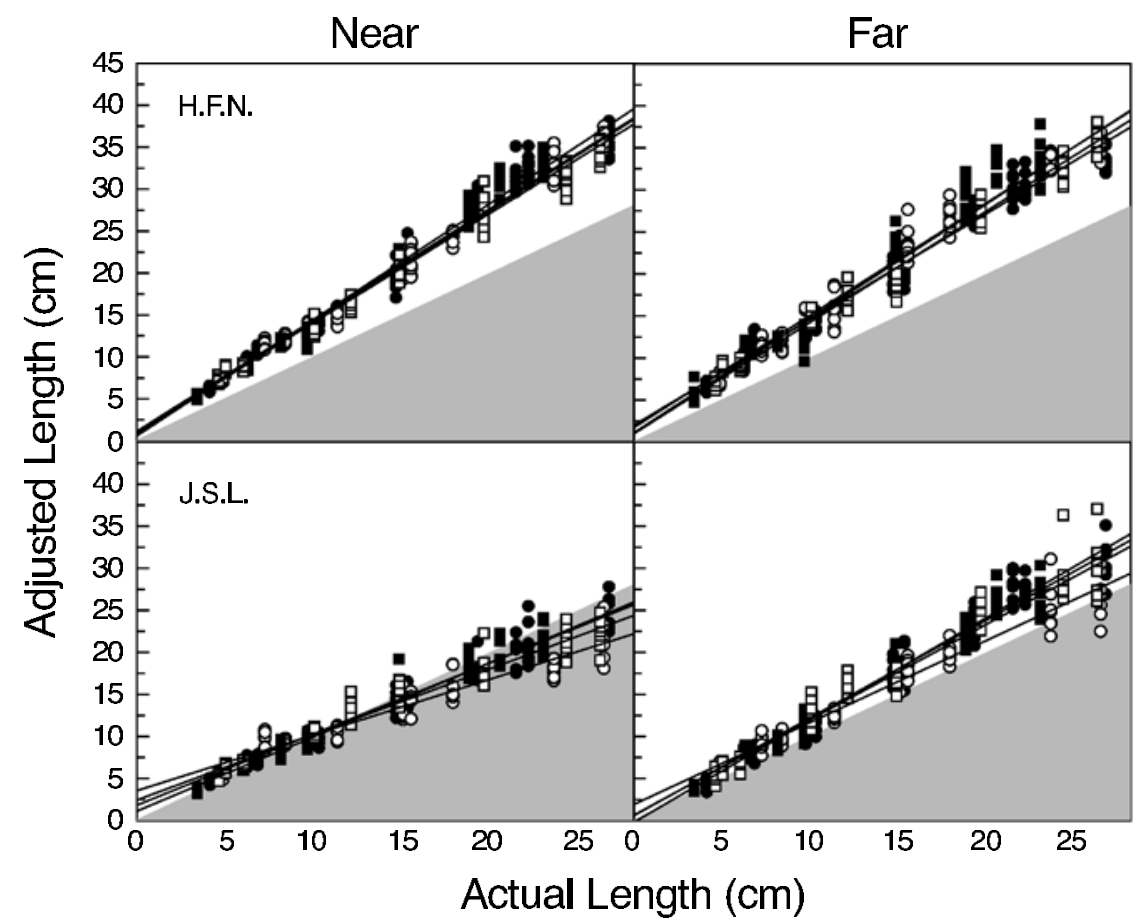

Figure 5. Results of Experiment 2 for the hyperbolic paraboloid for two representative observers (H.F.N. and J.S.L.). The observers' perceived lengths are plotted as functions of the actual surface lengths. In each graph, the boundary between the white and the gray areas indicates a slope of 1.0. Therefore, data points that fall within the white regions indicate overestimation, whereas data points that are plotted within the gray regions indicate underestimation. CW, clockwise; CCW, counterclockwise.

their mean) was $10.7 \%$, which was not statistically different from the previous average reliability for the same set of observers in Experiment $1[7.6 \% ; t(2)=2.711$, $p>.05]$.

\section{GENERAL DISCUSSION}

The results of all three experiments demonstrated a considerable human perceptual sensitivity to the differences in length that occur along curved object surfaces. About $95 \%$ of the variance in the observers' visual and motoric estimates of surface length could be accounted for by variations in the magnitudes of the lengths on the actual surfaces themselves. The precision of these judgments compares very favorably with that of other aspects of 3-D object shape. For example, observers in a study by Norman, Todd, and Phillips (1995) performed an orientation-matching task in which they adjusted a gauge figure until its 3-D orientation matched that of local regions on curved object surfaces that were defined by stereoscopic disparities, motion, shading, texture, and/or specular highlights (i.e., also a full-cue situation). Norman et al. (1995) found Pearson $r^{2}$ values of .95 for judgments of surface tilt and .46 for judgments of surface slant (see Figures 6 and 7 in Norman et al., 1995). Slant and tilt represent the two components of surface orientation (Gibson, 1950; Stevens, 1983). The precision of the observers' estimates of curved surface length in the present experiments was as high as those previously obtained for judgments of surface tilt and was greater than that obtained for judgments of surface slant. Thus, lengths along curved object surfaces seem to be encoded within the visual system at least as precisely as other aspects of 3-D object shape, such as surface orientation.

The present finding of precise judgments of surface length, obtained with bidirectionally curved surfaces (convex and concave hemispheres and a saddle-shaped surface), is similar to that obtained by Norman et al. (2000) for intervals along cylindrical surfaces (curvature in depth occurred in only one direction) and by Norman 
Table 4

Slopes of Best-Fitting Regression Lines for the Hyperbolic Paraboloid

\begin{tabular}{lcccc}
\hline & \multicolumn{4}{c}{ Length Orientation } \\
\cline { 2 - 5 } Observer & $\mathrm{V}$ & $\mathrm{H}$ & $\mathrm{CCW}$ & $\mathrm{CW}$ \\
\hline \multicolumn{5}{c}{ Near Distance } \\
H.F.N. & 1.392 & 1.339 & 1.333 & 1.310 \\
Y.L. & 1.283 & 1.234 & 1.242 & 1.294 \\
D.S. & 1.059 & 0.855 & 0.972 & 0.947 \\
E.D. & 1.064 & 0.829 & 0.952 & 0.964 \\
J.S.L. & 0.888 & 0.666 & 0.851 & 0.784 \\
& \multicolumn{5}{c}{ Far Distance } \\
H.F.N. & 1.266 & 1.370 & 1.345 & 1.332 \\
Y.L. & 1.222 & 1.240 & 1.179 & 1.288 \\
D.S. & 1.135 & 1.091 & 1.100 & 1.115 \\
E.D. & 1.135 & 0.733 & 0.846 & 0.907 \\
J.S.L. & 1.225 & 0.981 & 1.142 & 1.170 \\
\hline
\end{tabular}

Note-V, vertical; H, horizontal; $\mathrm{CCW}$, counterclockwise; $\mathrm{CW}$, clockwise.

et al. (1996, Experiment 4) for distances in "empty" space between real surface locations. All of these results indicate that observers' knowledge of distances on or between points on real-world surfaces is precise. This precise knowledge of distance, however, may be limited to full-cue situations in which observers directly view realworld surfaces. Using a discrimination paradigm, Norman et al. (1996, Experiment 2) found relatively poor performance for judgments of 3-D length for computergenerated figures defined by stereoscopic disparity and motion (Weber fractions ranged from $11.1 \%$ to $26.3 \%$ ). In contrast, the perception of two-dimensional length in the frontoparallel plane is almost always precise with Weber fractions of 1\%-3\% (Fechner, 1860/1966; Norman et al., 1996, Experiment 1; Weber, 1834/1978; see also Wundt, 1892/1901).

Despite the presence of high precision and reliability, the observers' perceptions of surface lengths in our study were often inaccurate. Furthermore, the patterns of these inaccuracies were specific to individual observers. Some observers tended to overestimate the surface lengths and perceive them to be (much) longer than they really were (e.g., H.F.N., Y.L., and L.M.H.), whereas other observers often underestimated the surface lengths and perceived them to be shorter than they were in reality (e.g., E.D. in Experiment 2 and at the far distance in Experiment 1, and J.S.L. at the near viewing distance in Experiment 2). The most common pattern throughout the present experiments was overestimation (see also Norman et al., 2000). This result is different from those in most previous studies in which the perception of distances in depth has been examined. In most of these studies, the observers estimated the size of in-depth intervals along a planar ground surface or table top; the most common pattern of perceptual distortion obtained in these experiments was underestimation, reflecting a perceptual compression of distances in depth (Baird \& Biersdorf, 1967; Gilinsky, 1951; Harway, 1963; Loomis et al., 1992; Norman et al., 1996; Wagner, 1985). Taken to- gether, a comparison of our present results that document overestimations (and those of Norman et al., 2000) with those of previous studies that showed underestimations strongly suggests that the perception of distances along the surfaces of curved objects follows a different set of principles than does the perception of distances between objects along planar or ground surfaces. If this is true, there is no consistent mathematical relationship between physical and perceived 3-D space that holds true in all circumstances (also see Cuijpers, Kappers, \& Koenderink, 2002).

In past research on the perception of distances in depth (e.g., Baird \& Biersdorf, 1967; Gilinsky, 1951; Harway, 1963; Norman et al., 1996), changes in viewing distance had consistent effects that were similar for most/all observers. This was not necessarily true in our present experiments. For example, in Experiment 1, the observers consistently perceived the surface lengths along the convex hemisphere to be longer at the near viewing distance and shorter at the far viewing distance. However, this was not the case for the surface lengths along either the concave hemisphere or the saddleshaped hyperbolic paraboloid. For the concave hemisphere, 2 observers perceived the surface lengths to be significantly longer at the far distance (L.M.H. and J.R.L.), whereas 1 observer (Y.L.) perceived the surface lengths to be significantly longer at the near distance (see Table 3). This inconsistency in the effects of viewing distance was also evident in Experiment 2: D.S. and J.S.L. perceived the lengths along the hyperbolic paraboloid to be significantly longer at the far viewing distance, whereas E.D. had the opposite pattern of results and perceived the same set of lengths to be longer at the near distance (see Table 5). This pattern of results across Experiments 1 and 2 reinforces the conclusion that there cannot be any single relationship between physical space and perceived space that is consistent across observers, since the judgments of some observers increase with increases in viewing distance, whereas the judgments of other observers decrease. Large individual differences have also been observed in earlier studies of the perception of 3-D shape and depth (see, e.g., Todd \& Norman, 2003). These effects of viewing distance on the perception of length (i.e., failures of constancy) are especially

Table 5

Effects of Distance for the Hyperbolic Paraboloid

\begin{tabular}{lccrrr}
\hline & \multicolumn{2}{c}{ Slope } & & & \\
\cline { 2 - 5 } & $\begin{array}{c}\text { Near } \\
\text { Observer }\end{array}$ & $\begin{array}{c}\text { Far } \\
\text { Judgments }\end{array}$ & & & \\
Judgments & Change $\%$ & \multicolumn{1}{c}{$t$} & \multicolumn{1}{c}{$p$} \\
\hline H.F.N. & 1.340 & 1.331 & -0.7 & 0.360 & .737 \\
Y.L. & 1.263 & 1.231 & -2.5 & 2.106 & .103 \\
D.S. & 0.964 & 1.110 & +15.1 & -6.023 & .004 \\
J.S.L. & 0.810 & 1.143 & +41.1 & -17.462 & $<.001$ \\
E.D. & 0.961 & 0.866 & -9.9 & 3.972 & .017 \\
\hline
\end{tabular}

Note-Results of the regression analysis that evaluated the effects of viewing distance for the hyperbolic paraboloid used in Experiment 2. All $d f \mathrm{~s}=4$, except for that of J.S.L., where $d f=3$. 


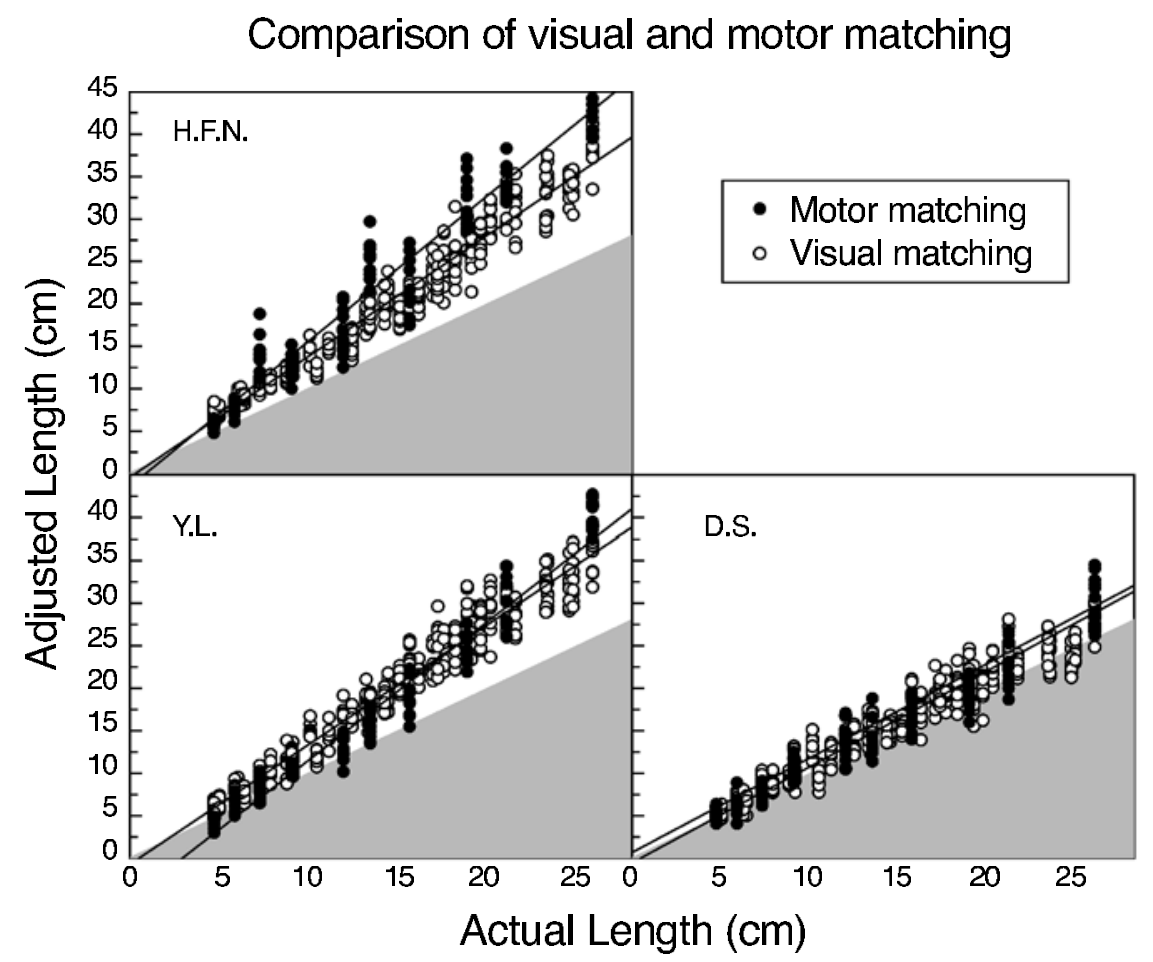

\begin{abstract}
Figure 6. Results of Experiment 3 for the convex hemisphere for all the observers. The observers' perceived lengths are plotted as functions of the actual surface lengths. In each graph, the boundary between the white and the gray areas indicates a slope of 1.0. Therefore, data points that fall within the white regions indicate overestimation, whereas data points that are plotted within the gray regions indicate underestimation.
\end{abstract}

intriguing, because in all of our experiments, the surface lengths were viewed at very short viewing distances that were always less than $2 \mathrm{~m}$. Traditionally (see, e.g., Gulick \& Lawson, 1976, pp. 250-252), human observers have been considered to possess good retinal and extraretinal information (accommodation, accommodative blur, and convergence) about viewing distances less than $2 \mathrm{~m}$. Our stimuli were viewed from short distances, a situation in which accommodation and convergence should have been effective (full-cue viewing of actual physical surfaces from $1.8 \mathrm{~m}$ or less) sources for providing the information about absolute depth that is needed to correctly scale the binocular disparities and, thus, permit length constancy. Nevertheless, changing the viewing distances in our experiments led to alterations in the perceived surface lengths by most of the observers. Perhaps accommodation and convergence have less of a role to play in the perception of 3-D spatial intervals than has traditionally been suspected.

The results of Experiments 1 and 2 also revealed an anisotropy of orientation of the surface lengths. Across those experiments, the vertically oriented lengths appeared to be $8.6 \%$ longer than physically equivalent horizontal lengths. This is especially interesting since the viewing situation was full cue. The observers had a wealth of optical information upon which to support their perceptions of surface length: binocular disparity, motion parallax, texture gradients, image shading, and so forth. Nevertheless, changes in the orientation in the retinal image of the surface intervals led to substantial changes in their perceived lengths. This effect of orientation is even larger than that typically obtained in studies of the horizontal-vertical illusion (7.2\%; Finger \& Spelt, 1947).

In this study, we evaluated observers' perceptions of length along curved surfaces with positive and negative Gaussian curvature (Experiments 1 and 2, respectively). We used these two types of surfaces because the results of differential geometry have revealed that these are the only two kinds of generic surface regions that smoothly curved objects possess (see, e.g., Hilbert \& Cohn-Vossen, 1952; Koenderink, 1990; Koenderink \& van Doorn, 1982). In general, the observers perceived the surface lengths similarly for all of the surfaces that we used. Nevertheless, there were some interesting differences. Note, for example, that the judgments of Y.L. were greatly affected by changing the curvature of the hemisphere from convex to concave (compare Tables 1 and 2). In fact, her estimates of the magnitudes of the surface lengths dropped by amounts up to $40 \%$ when the direction of curvature was changed from convex to concave. After the experiments were completed, she commented that 


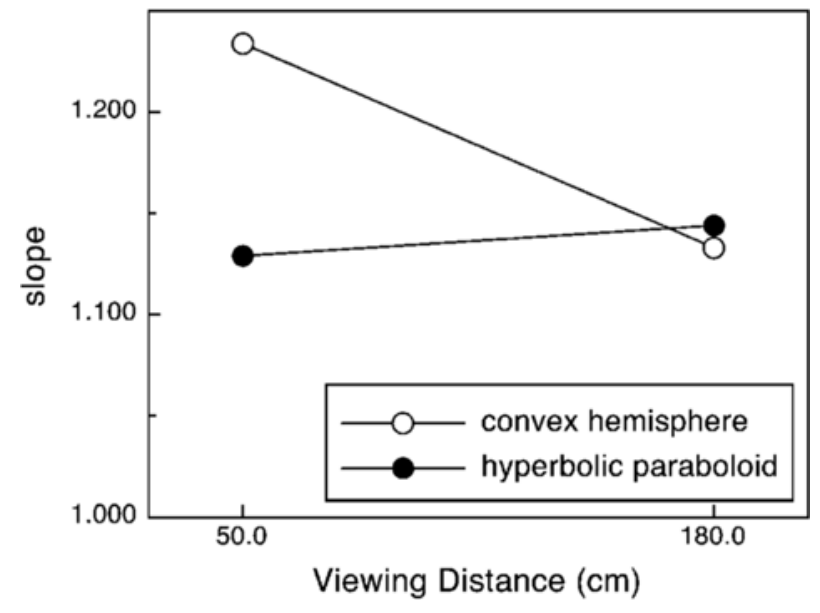

Figure 7. A plot comparing the results of Experiments 1 and 2, showing that changes in viewing distance have different effects on the perception of length that depend on the type of surface curvature.

the concave hemisphere "appeared" to have much less front-to-back depth and curvature than the convex hemisphere. The reduction in the perceived surface lengths with the concave hemisphere is consistent with her phenomenological observations. Other differences also emerged between the different surface types. For example, consider the results shown in Figure 7. One can see in this figure that there were significantly different effects of viewing distance upon the observers' estimates of the surface lengths along the convex hemisphere and the hyperbolic paraboloid $[F(1,3)=13.2, p=.036$, on the basis of the data of the 4 observers who judged both surfaces]. Whereas all the observers perceived the lengths of the surface intervals along the convex hemisphere to decrease with increases in viewing distance, there was no such consistent effect for distances along the surface of the hyperbolic paraboloid.

"Ordinary" human observers typically believe quite strongly that their perceptions of size, distance, and length are accurate. Furthermore, they feel that their perceptions of length demonstrate constancy and that they are unaffected by changes in viewing distance, orientation, and so forth. Probably, this is related to the ease with which these judgments can be performed: Judgments and comparisons of length do not seem to be difficult tasks, especially when compared with other psychological tasks that require effort (e.g., listing the contents of shortterm memory backward or a large number of other such tasks). Descartes (1628/1955), in referring to the perception of length and depth, remarked that "there is nothing more easily perceived" (p. 57). This perceptual ease masks the fact that the successful recovery of "correct" 3-D distances and lengths from optical information, such as binocular disparity, motion, texture gradients, image shading, and so forth, is a computationally difficult task that may be perceptually impossible for human observers to perform with both precision and accuracy in all cir- cumstances. The results of our experiments show that human perception of length along object surfaces, although precise, is generally inaccurate. Observers' estimates of surface length are influenced by many factors, such as the type and curvature of the surface (flat vs. curved vs. the type of curvature), the orientation of the surface (see Norman et al., 2000), the orientation of the lengths on the surface (Experiments 1 and 2), the distance to the surface (Experiments 1 and 2), and so forth. In addition, the patterns of results that are obtained are often unique to each individual observer (i.e., there are large individual differences). Nevertheless, each observer's perceptions of surface length are reliable and consistent over time; the observers exhibit the same patterns of perceptual distortion for days and weeks, if not for years. Finally, our results demonstrate that observers' judgments of surface length show the same inaccuracies whether the length-matching judgments are performed visually or motorically (Experiment 3 ). It is probably a consequence of these many perceptual inaccuracies and failures of constancy that we so frequently come to rely on rulers and other measurement tools when we need to accurately estimate distances along environmental surfaces.

\section{REFERENCES}

Aristotle (trans., 1984). On the soul. In J. Barnes (Ed.), The complete works of Aristotle (Vol. 1, pp. 641-692). Princeton, NJ: Princeton University Press.

BAIRD, J. C., \& BIERSDORF, W. R. (1967). Quantitative functions for size and distance judgments. Perception \& Psychophysics, 2, 161-166.

CuiJPers, R. H., Kappers, A. M. L., \& Koenderink, J. J. (2002). Visual perception of collinearity. Perception \& Psychophysics, 64, 392-404.

DESCARTES, R. (1955). Rules for the direction of the mind. In E. S. Haldane \& G. R. T. Ross (Eds. and Trans.), The philosophical works of Descartes (Vol. 1, pp. 1-77). New York: Dover. (Original work published 1628)

FECHNER, G. T. (1966). Elements of psychophysics (Vol. 1; H. E. Adler, Trans.). New York: Holt, Rinehart, \& Winston. (Original work published 1860)

FINGER, F. W., \& SPELT, D. K. (1947). The illustration of the horizontalvertical illusion. Journal of Experimental Psychology, 37, 243-250.

GiBson, J. J. (1950). The perception of the visual world. Boston: Houghton Mifflin.

Gilinsky, A. S. (1951). Perceived size and distance in visual space. Psychological Review, 58, 460-482.

GoGEL, W. C. (1960). The perception of a depth interval with binocular disparity cues. Journal of Psychology, 50, 257-269.

Graham, C. H. (1951). Visual perception. In S. S. Stevens (Ed.), Handbook of experimental psychology (pp. 868-920). New York: Wiley.

Gulick, W. L., \& Lawson, R. B. (1976). Human stereopsis: A psychophysical approach. New York: Oxford University Press.

HARWAY, N. I. (1963). Judgment of distance in children and adults. Journal of Experimental Psychology, 65, 385-390.

Hilbert, D., \& Cohn-Vossen, S. (1952). Geometry and the imagination. New York: Chelsea.

JULESZ, B. (1971). Foundations of cyclopean perception. Chicago: University of Chicago Press.

Koenderink, J. J. (1990). Solid shape. Cambridge, MA: MIT Press. KoEnderink, J. J., \& VAN DOORN, A. J. (1982). The shape of smooth objects and the way contours end. Perception, 11, 129-137.

Loomis, J. M., DA Silva, J. A., Fujita, N., \& Fukusima, S. S. (1992). Visual space perception and visually directed action. Journal of Experimental Psychology: Human Perception \& Performance, 18, 906921.

Norman, J. F., LAPPIN, J. S., \& Norman, H. F. (2000). The perception 
of length on curved and flat surfaces. Perception \& Psychophysics, 62, 1133-1145.

NoRmAn, J. F., \& TODD, J. T. (1998). Stereoscopic discrimination of interval and ordinal depth relations on smooth surfaces and in empty space. Perception, 27, 257-272.

Norman, J. F., Todd, J. T., Perotti, V. J., \& Tittle, J. S. (1996). The visual perception of three-dimensional length. Journal of Experimental Psychology: Human Perception \& Performance, 22, 173-186.

Norman, J. F., TodD, J. T., \& Phillips, F. (1995). The perception of surface orientation from multiple sources of optical information. Perception \& Psychophysics, 57, 629-636.

Pollock, J. (1978).Jackson Pollock: A catalogue raisonné of paintings, drawings, and other works. New Haven, CT: Yale University Press.

Rogers, B., \& GRAHAM, M. (1979). Motion parallax as an independent cue for depth perception. Perception, 8, 125-134.

Ste vens, K. A. (1983). Slant-tilt: The visual encoding of surface orientation. Biological Cybernetics, 46, 183-195.
TODD, J. T., \& Norman, J. F. (2003). The visual perception of 3-D shape from multiple cues: Are observers capable of perceiving metric structure? Perception \& Psychophysics, 65, 31-47.

Wagner, M. (1985). The metric of visual space. Perception \& Psychophysics, 38, 483-495.

Weber, E. H. (1978). The sense of touch (H. E. Ross, Trans.). New York: Academic Press. (Original work published 1834)

WoOdWORTH, R. S., \& SCHLOSBERG,H. (1954). The perception of form. In R. S. Woodworth \& H. Schlosberg, Experimental psychology (pp. 403-427). New York: Holt.

WUNDT, W. (1901). Lectures on human and animal psychology (J. E. Creighton \& E. B. Titchener, Trans.). New York: Macmillan. (Original work published 1892)

(Manuscript received November 12, 2002; revision accepted for publication June 2, 2003.) 